\title{
Effect of resident handoffs on length of hospital and intensive care unit stay in a neurosurgical population: a cohort study
}

\author{
Elizabeth N. Kuhn, MD, ${ }^{1}$ Matthew C. Davis, MD, ${ }^{1}$ Bonita S. Agee, PhD, MPH, ${ }^{1}$ Robert A. Oster, PhD, ${ }^{2}$ \\ and James M. Markert, MD, MPH ${ }^{1}$
}

\begin{abstract}
1Department of Neurosurgery and ${ }^{2}$ Division of Preventive Medicine, Department of Medicine, University of Alabama at Birmingham, Alabama
\end{abstract}

\begin{abstract}
OBJECTIVE Handoffs and services changes are potentially modifiable sources of medical error and delays in transition of care. This cohort study assessed the relationship between resident service handoffs and length of stay for neurosurgical patients.
\end{abstract}

METHODS All patients admitted to the University of Alabama at Birmingham neurosurgical service between July 1 , 2012, and July 1, 2014, were retrospectively identified. A service handoff was defined as any point when a resident handed off coverage of a service for longer than 1 weekend. A conditional probability distribution was constructed to adjust length of stay for the increasing probability of a random handoff. The Student t-test and ANCOVA were used to assess relationships between resident service handoffs and length of hospital stay, adjusted for potential confounders.

RESULTS A total of 3038 patients met eligibility criteria and were included in the statistical analyses. Adjusted length of hospital stay (5.32 vs 3.53 adjusted days) and length of ICU stay (4.38 vs 2.96 adjusted days) were both longer for patients who experienced a service handoff, with no difference in mortality. In the ANCOVA model, resident service handoff remained predictive of both length of hospital stay $(p<0.001)$ and length of ICU stay $(p<0.001)$.

CONCLUSIONS Occurrence of a resident service handoff is an independent predictor of length of hospital and ICU stay in neurosurgical patients. This finding is novel in the neurosurgical literature. Future research might identify mechanisms for improving continuity of care and mitigating the effect of resident handoffs on patient outcomes.

http://thejns.org/doi/abs/10.3171/2015.7.JNS15920

KEY WORDS neurosurgery; patient handoff; patient outcome; length of stay

$\mathrm{N}$ EUROSURGEONS encounter many challenges in delivering high-quality patient care within the restrictions of resident duty hours, including some unique hurdles not faced by other surgical and medical disciplines. These include smaller numbers of residents per program, large censuses, highly complex cases and critically ill patients, and dependence on serial neurological examinations. Following the initiation of duty-hour restrictions by the Accreditation Council for Graduate Medical Education (ACGME) in 2003 and revisions in 2011, it was quickly recognized that surgical residents often needed to work more than 80 hours per week to complete clinical and operative duties. The ACGME consequently allowed residency programs to apply for extensions of up to 88 hours per week, and neurosurgery has been the only specialty granted these extensions to date. ${ }^{4}$

Despite the extension of 88 hours per week, the ability of neurosurgical residents to provide quality care while receiving adequate training has been questioned. ${ }^{18,23}$ Norby et al. found that following the implementation of ACGME duty-hour restrictions in neurosurgery, postoperative complications and mean hospital charges increased, without improvement in outcomes. ${ }^{28} \mathrm{Hoh}$ et al. demonstrated that the duty-hour restriction era correlated with an increased risk of postoperative complications and no change in mortality for neurosurgical trauma patients. ${ }^{21}$ Babu et al. re-

ABBREVIATIONS ACGME = Accreditation Council for Graduate Medical Education; DRG = diagnosis-related group; FIRST = Flexibility In duty hour Requirements for Surgical Trainees; LOS = length of stay.

SUBMITTED April 25, 2015. ACCEPTED July 15, 2015.

INCLUDE WHEN CITING Published online December 18, 2015; DOI: 10.3171/2015.7.JNS15920. 
ported similar results for patients undergoing brain tumor, cerebrovascular, and spine procedures. ${ }^{4-6}$ Additionally, the ACGME requirements mandate that residents have a break period between shifts, which necessitates an increased frequency in number of handoffs between shifts and impairs continuity of care. ${ }^{24}$

A handoff involves some form of verbal and written communication between providers as they change shifts. The goal of an effective handoff is to safely transition the primary responsibility of patient care to another person. ${ }^{10}$ Although some specialties, including internal medicine and pediatrics, have studied handoffs in an effort to identify problems and improve efficiency in light of duty-hour restrictions, ${ }^{14,22}$ there is still a need for high-quality handoff outcomes studies, especially in surgical specialties. ${ }^{30}$ Handoffs and services changes can be particularly challenging in neurosurgery programs due to the low number of residents employed per year, large censuses, and importance of subjective measures, especially the neurological examination.

Serial neurological examinations are the substrate of neurosurgical decision making; however, studies have shown that the interrater reliability of those examinations is imperfect..$^{33}$ Elicitable signs, including strength, reflexes, and sensation, have a wide range of reported reliability, from poor to substantial $(\kappa 0.15-0.77){ }^{33}$ Handoffs create the potential for subtle differences in these subjective measures to be inadequately conveyed from one provider to the next, which could prevent a physician from noting early changes in neurological status and thus delay intervention. Additionally, when a new resident takes over a large service after a rotation change, there is an inevitable lag time before the resident becomes knowledgeable about each patient. Despite these potential sources of error, little attention has been given to the effect of handoffs or strategies for improvement of the handoff within the neurosurgical community. ${ }^{4}$

Discontinuity in patient care resulting from handoffs may contribute to in-hospital complications, prolonged length of stay (LOS), and increased costs. ${ }^{27,34}$ This has been demonstrated in both surgical and medical specialties in the settings of shift change, weekends, and short versus long call periods. ${ }^{8,31,35}$ To our knowledge, there have been no studies in the neurosurgical community evaluating the relationship between resident handoffs and hospital LOS. Even if an extended hospital LOS does not result directly from medical error or complication, it leads to increased use of medical resources. In this case-control study, we compared the overall hospital LOS, ICU LOS, mortality, and readmission rates among patients who did or did not experience a resident handoff during their stay.

The hypothesis of this study was that patients have longer LOS when their stay includes a resident handoff than when there is no resident handoff. We suspect that following a resident handoff, as the new resident is becoming familiar with the patients on the service, there may be a delay in discharging patients. Although there may not be a medical error or complication per se, we anticipated that LOS would be longer secondary to this adjustment period. The purpose of this study was to better understand the effect of resident handoffs on LOS in neurosurgical patients.

\section{Methods \\ Patient Selection and Sample Size Determination}

All patients admitted to the neurosurgical service at the University of Alabama at Birmingham between July 1, 2012, and July 1, 2014, were retrospectively identified (in February 2015) from billing records. This included patients who were admitted from the emergency room, transferred from another hospital or another service within our hospital, admitted after surgery, and admitted for same-day surgery. Only patients for whom a neurosurgeon was the primary attending physician of record were included. Sample size was determined by the availability of resident service schedules, which were not preserved prior to 2012. The follow-up period for patients ended with hospital discharge.

\section{Data Collection}

Data collected for this study included patient demographic information, surgical procedure, hospital LOS, ICU LOS, coincident resident service change, source of admission (home, clinic, emergency department, and so on), whether hospital stay spanned a weekend, admitting/ discharging attending physician, admitting unit, discharge disposition, mortality, readmission within 30 days, and admission day of the week. Data were collected retrospectively via chart review. There were no missing data.

The exposure of interest was occurrence of a resident service handoff during admission. A service handoff was defined as any point when a resident handed off coverage of a service for longer than 1 weekend. Handoffs occurred when residents transitioned between attending services, during vacations, and other absences. On average, a service handoff occurs every 30 days for all attending neurosurgeons. Patients with an LOS of 0 or 1 day were excluded due to no chance of a service handoff according to our definition. As a result, all patients who underwent outpatient surgery were excluded from statistical analyses. In addition, patients with a hospital stay longer than 30 days were excluded because resident handoff would have been guaranteed. Outcomes of interest were overall hospital LOS, ICU LOS, and mortality. Potential confounders included age, sex, admission status, source of admission, and admission day of the week.

Additionally, a matched pairs cohort analysis was done. Two subsets were examined: patients undergoing cervical fusion (diagnosis-related groups [DRGs] 471-473) and patients undergoing pituitary surgery (DRG 614). These subsets were chosen because they are performed with high frequency at our institution and are likely to contain similar groups of patients. The exposure of interest was a resident handoff. Groups were matched by DRG, age (within 3 years), and sex. The outcome of interest was adjusted LOS.

\section{Statistical Analysis}

Patients with a resident handoff $(n=525)$ were compared with patients without a resident handoff $(n=2513)$ with respect to hospital and ICU LOS, mortality, readmission rate, admission status, age, sex, discharge status, and weekend stay. First, Student t-tests were performed to determine the 
independent effect of age, resident handoff, weekend stay, and ICU admission on hospital and ICU LOS. Those variables significantly associated with the outcome of interest (i.e., hospital LOS, ICU LOS, and mortality) were included in the respective ANCOVA. Patients who had a longer hospital LOS had an increased nonrandom chance of experiencing a resident service handoff purely on the basis of handoffs occurring, on average, every 30 days. To adjust for this, a conditional probability distribution model was constructed. Such a model provides an adjusted distribution of hospital LOS (or ICU LOS) given the incremental risk of experiencing a resident handoff with increasing LOS. In other words, the conditional probability is an update of the probability of an event (e.g., resident handoff) based on new information (e.g., LOS). The following formula, a variation of the Bayes theorem, was used. It was derived from the formula described most famously by the Monty Hall problem: ${ }^{19}$ Adjusted LOS $=$ LOS - LOS*(LOS/30). The same formula ${ }^{7}$ was applied to ICU LOS.

The assumptions for ANCOVA were tested on the data to ensure the appropriateness of the analysis. Normality of distribution of LOS was confirmed using the KolmogorovSmirnov test. The relationship between the covariates and dependent variable was not influenced by the independent variable, thus confirming the homogeneity of slopes assumption. The assumptions of independence and normality were met. The assumption of homogeneity of variance was tested using Levene's test and the nonsignificant results $(\mathrm{F}$ ratio $=0.19 ; \mathrm{p}=0.66)$ did not violate the assumption of homogeneity of variance.

Paired 1-way Student t-tests were performed to compare adjusted LOS between groups in the matched pairs subset analyses. All of the statistical analyses were performed using JMP, version 11.0.0 (SAS Institute, Inc.).

\section{Mitigation of Bias}

Because this is a retrospective cohort study, the following steps were taken to mitigate the potential for bias. The individual(s) collecting data were blinded to presence/absence of coincident resident handoff. To decrease selection bias, a single population, restricted to all elective neurosurgical procedures performed on patients admitted to University of Alabama at Birmingham Hospital during the specified time period, was used to select both cohorts. To minimize recall bias, none of the variables analyzed in this study relied on the subject's or clinician's memory; all data were derived expressly from medical and billing records. As such, information error would be a function of nondifferential misclassification and would have the same likelihood of errors and omissions in these records.

This study was approved by the institutional review board of the University of Alabama at Birmingham. This manuscript was prepared following the STrengthening the Reporting of OBservational studies in Epidemiology (STROBE) checklist for cohort studies.

\section{Results \\ Study Population}

During the study period, 5531 patients were admitted to the neurosurgical service, of whom 3133 patients met inpatient criteria (LOS > 1 day). Ninety-five patients were excluded due to LOS beyond 30 days. This resulted in a final study population of 3038 patients who were included in statistical analyses; 525 patients (17.3\%) experienced a handoff, and 2513 patients $(82.7 \%)$ did not experience a handoff. The mean patient age was 55.2 years and $51.4 \%$ were women.

\section{Mortality and 30-Day Readmission Rates}

Eighty-four patients (2.8\%) died during their hospitalization. Eighteen deaths were among patients who experienced a resident handoff; the remaining 66 deaths were among patients who did not experience such a handoff. Bivariate analysis indicated that resident change was not associated with mortality $(\mathrm{p}=0.3)$. However, mortality was associated with age $\left(\chi^{2}=17.6 ; \mathrm{p}<0.001\right)$, admission to ICU ( $8.6 \%$ vs $\left.0.71 \% ; \chi^{2}=135.8 ; \mathrm{p}<0.001\right)$, and day of week of admission $\left(\chi^{2}=27.4 ; \mathrm{p}<0.001\right)$. Admission on a Saturday carried the highest risk of mortality (7.5\%) compared with Friday, which had the lowest risk of mortality $(1.4 \%)$.

Overall, $11.3 \%$ of patients were readmitted within 30 days. Among patients who experienced a resident handoff, the 30-day readmission rate was $9.1 \%$, whereas the 30 day readmission rate was $11.7 \%$ among patients without a resident handoff. Bivariate analysis indicated that resident change was not associated with 30-day readmission ( $\mathrm{p}=$ $0.09)$.

\section{Overall LOS}

The mean LOS for patients who experienced a service handoff was 10.7 days versus 5.0 days for patients who did not experience a handoff. The mean adjusted LOS for patients who experienced a service handoff was 5.3 adjusted days versus 3.5 adjusted days for patients who did not experience a handoff (Table 1). An ANCOVA model was constructed using the variables of resident handoff, weekend stay, admission to ICU, and age, which were all significantly associated with adjusted LOS on bivariate analysis ( $\mathrm{p}<0.01)$. Resident service handoff $(\mathrm{p}<0.001)$, weekend stay $(\mathrm{p}<0.001)$, age $(\mathrm{p}=0.04)$, and admission to ICU $(\mathrm{p}<0.001)$ all remained predictive of adjusted LOS in the multivariate model (Table 2).

Post hoc analyses were then performed to assess the relationship between timing of handoff and overall LOS. Considering only those patients who experienced a handoff during the first 3 hospital days, those patients with a handoff had significantly longer adjusted LOS than those without a handoff (4.6 vs 3.5 adjusted days; $\mathrm{p}<0.0001$ ). Patients who experienced a handoff during their ICU stay had a longer adjusted LOS than patients who did not (5.7 vs $5.4 ; \mathrm{p}=0.048$ ).

\section{ICU LOS}

The mean ICU LOS for patients who experienced a service handoff was 6.9 days versus 3.8 days for patients who did not experience a handoff. Adjusted ICU LOS for patients who experienced a handoff was 4.4 adjusted days versus 3.0 adjusted days for patients who did not experience a handoff (Table 1). An ANCOVA model was con- 
TABLE 1. Comparison of demographic and clinical data between cohorts*

\begin{tabular}{lccc}
\hline \multicolumn{1}{c}{ Variable } & Resident Handoff, $n=525$ & No Resident Handoff, $n=2513$ & $p$ Value† \\
\hline Age in yrs, mean (SD) & $56.3(16.4)$ & $55.0(16.1)$ & 0.94 \\
\hline Female, $\%$ & 51.2 & 51.5 & 0.96 \\
\hline Admission to ICU, $\%$ & 45.0 & 22.0 & $<0.001$ \\
\hline Stay including a weekend, $\%$ & 96.7 & 57.5 & $<0.001$ \\
\hline Adjusted hospital LOS in days, mean (SD) & $5.32(1.76)$ & $3.53(1.78)$ & $<0.001$ \\
\hline Adjusted ICU LOS in days, mean (SD) & $4.38(2.00)$ & $2.96(1.56)$ & $<0.001$ \\
\hline Mortality, \% & 3.4 & 2.6 & 0.31 \\
\hline 30-day readmission, $\%$ & 9.1 & 11.7 & 0.09 \\
\hline
\end{tabular}

* Student t-test was used for comparison of means; chi-square was used for comparison of proportions.

$\dagger$ Statistically significant at $p<0.05$.

structed using the variables resident handoff and weekend stay, both shown to be significantly associated with adjusted ICU LOS on bivariate analysis ( $p<0.001)$. Resident change and weekend stay remained statistically significant predictors of adjusted ICU LOS in a multivariate model incorporating both variables $(\mathrm{p}<0.001)$.

Post hoc analyses were then performed to assess the relationship between timing of handoff and ICU LOS. Considering only those patients who experienced a handoff during the first 3 hospital days, those patients with a handoff had a significantly longer adjusted ICU LOS compared with those patients without a handoff (3.92 vs 2.97 adjusted days; $\mathrm{p}<0.0001)$. Patients who experienced a handoff during their ICU stay had significantly longer adjusted ICU LOS compared with those who did not (5.4 vs 3.4 adjusted days; $\mathrm{p}<0.0001$ ).

\section{Matched Pairs Comparisons}

We performed additional subset analyses using matched pairs design for 2 common procedures, namely pituitary surgery and cervical fusion. There were 18 matched pairs among patients undergoing cervical fusion. Among these patients, those who experienced a resident handoff had a significantly longer adjusted LOS compared with those with no resident handoff (5.25 vs 4.21 adjusted days; $\mathrm{p}=$ $0.027)$. There were 10 matched pairs among patients undergoing pituitary surgery. These patients also had a significantly longer adjusted LOS when a resident handoff occurred compared with no resident handoff (3.92 vs 2.92 adjusted days; $\mathrm{p}=0.028$ ).

\section{Discussion}

We completed a comprehensive analysis of the effect of resident handoffs on hospital and ICU LOS for a neurosurgical service at a busy, tertiary care teaching hospital. There are several limitations to this study. Data were retrospectively reviewed, with the inherent limitation of being nonrandomized. A randomized trial on the specific issue of whether a resident handoff affects hospital or ICU LOS is logistically impossible to conceive. However, randomized controlled trials in which the duty-hour restrictions are relaxed, such as the Flexibility In duty hour Requirements for Surgical Trainees (FIRST) trial, may provide important insights. The FIRST trial is an ongoing cluster-randomized trial that randomizes general surgery residency programs to either a control group with continued adherence to current requirements or an intervention group with increased flexibility of duty-hour restrictions for interns (http://www.thefirsttrial.org). The outcomes of this study include the traditional metrics of death, morbidity, and complications along with resident perceptions, resident case volume, American Board of Surgery In-Training Examination scores, and program director surveys. Although the frequency of handoffs is not specifically measured in the FIRST trial, it can be assumed that hand-

TABLE 2. Bivariate and multivariate analyses*

\begin{tabular}{|c|c|c|c|c|c|c|c|c|c|}
\hline \multirow[b]{3}{*}{ Variable† } & \multicolumn{3}{|c|}{ Adjusted Hospital LOS } & \multicolumn{3}{|c|}{ Adjusted ICU LOS } & \multicolumn{3}{|c|}{ Mortality } \\
\hline & \multirow{2}{*}{$\begin{array}{c}\text { Bivariate } \\
\text { Analysis } \\
\text { ( } p \text { value) } \ddagger\end{array}$} & \multicolumn{2}{|c|}{ Multivariate Analysis } & \multirow{2}{*}{$\begin{array}{c}\text { Bivariate } \\
\text { Analysis } \\
\text { ( } p \text { value }) \ddagger\end{array}$} & \multicolumn{2}{|c|}{ Multivariate Analysis } & \multirow{2}{*}{$\begin{array}{c}\text { Bivariate } \\
\text { Analysis } \\
\text { ( } p \text { value) } \ddagger\end{array}$} & \multicolumn{2}{|c|}{ Multivariate Analysis } \\
\hline & & Coefficient & p Valuef & & Coefficient & p Valuef & & Coefficient & p Valuef \\
\hline Age & 0.002 & 0.0033 & 0.04 & 0.17 & NA & NA & $<0.001$ & 0.00063 & 0.0004 \\
\hline Resident handoff & $<0.001$ & 0.84 & $<0.001$ & $<0.001$ & 0.95 & $<0.001$ & 0.3 & NA & NA \\
\hline Weekend stay & $<0.001$ & 2.0 & $<0.001$ & $<0.001$ & 1.37 & $<0.001$ & 0.01 & 0.0066 & 0.3 \\
\hline Admission to ICU & $<0.001$ & 0.67 & $<0.001$ & NA & NA & NA & $<0.001$ & 0.080 & $<0.001$ \\
\hline
\end{tabular}

$\mathrm{NA}=$ not applicable.

* Student t-test was used for bivariate analysis of dichotomous independent variables and continuous dependent variables; chi-square was used for comparison of proportions.

† The ANCOVA model includes the independent variables age, resident handoff, weekend stay, and admission to ICU as predictors of hospital LOS and ICU LOS.

$\ddagger$ Statistically significant at $p<0.05$. 
offs will decrease in the intervention arm compared with the control arm. However, the specific impact of handoffs will be difficult to quantify because there are numerous other factors, such as resident fatigue, being altered in this trial that may impact their outcomes of study.

Studies in neurosurgery, such as those by Babu and colleagues, ${ }^{4-6}$ report an increase in complications coincident with the duty-hour restriction era. High-level meta-analyses of the surgical literature show no effect on patient mortality and morbidity in the duty-hour restriction era. ${ }^{2,25}$ Especially among high-acuity patients and surgical subspecialties, there is evidence that duty-hour restrictions increase morbidity. ${ }^{13,17,21,26}$ A study of surgical ICUs showed that increased complications, re-intervention, and ICU readmission resulted from increased fragmentation of care. ${ }^{9}$ Looking specifically at a neurosurgical population, Dumont et al. reported increased morbidity, especially avoidable morbidity, but decreased mortality after institution of the 2003 duty-hour restrictions. ${ }^{13}$ These data were collected prospectively by neurosurgery residents and attending physicians caring for the patients under study. Although this study measures specific sources of morbidity along with mortality, it does not control for shifts in the types of neurosurgical disorders seen at that hospital over time. The literature remains inconclusive on the impact of dutyhour restrictions on patient outcomes in neurosurgery. ${ }^{2}$

In our study, the outcomes of interest focused on hospital and ICU LOS, but specific complications were not measured or recorded. LOS and mortality were chosen as our outcomes of interest because they are reliable and uncontestable. Hospital and ICU LOS may reasonably be considered a proxy for significant complications. Differences in patient outcomes among hospitals have been shown to stem not from differential complication rates but from a failure to recognize and appropriately resuscitate patients with those complications. ${ }^{15,16}$ Furthermore, LOS may be prolonged even in the absence of complications. For example, if physical therapy and/or social work are not promptly involved, there may be a delay until the patient can be safely discharged. Although this would not constitute a complication per se, it does increase use of health care resources. In the modern era of health care efficiency, this will be increasingly scrutinized.

Studies investigating postoperative adverse events are themselves complicated by inaccuracies in the medical record, inconsistent coding, and absent data. Temporally coincident with the implementation of duty-hour restrictions in 2003 were the widespread use of electronic medical records and dramatic changes in coding and billing practices. Electronic health records may increase the recording of complications, leading to a potentially significant missing data bias for the electronic record era compared with the paper record era. Furthermore, surgical coding is highly prone to subjectivity, variability, and error. A large, recently published study of the accuracy of clinical coding in surgery revealed that a coding audit led to a change in $51 \%$ of patients, including changes in morbidity level and frequency of complications. ${ }^{29}$ Many published studies on complications and their relationship to resident handoffs or duty hours use large administrative databases, which are well known to contain inaccuracies of data acquisi- tion; ${ }^{4-6,21}$ obviously, clinically applicable conclusions can only be made from data that are accurately recorded.

Our study reports that hospital stays including a weekend, in addition to resident handoffs, were significantly associated with longer hospital and ICU LOS. There are probably multiple reasons for this. First, patients admitted on the weekend are an inherently different patient population than those admitted during the week. Admissions during the week include both elective admissions for surgery and nonelective admissions, whereas admissions on the weekend include almost exclusively nonelective admissions. There may be a larger proportion of high-acuity patients, who might have longer hospitalizations than their less-ill counterparts. Certain resources are less available over the weekend, such as electroencephalography interpretation, diagnostic ultrasound, physical and occupational therapy, and social work, all of which have the potential to adversely impact hospital LOS. Finally, resident coverage varies over the weekend. In some cases, the primary resident continues to provide care over the weekend without any discontinuity; in other cases, the opposite is true. The sum of all these factors is unclear, although many hypothesize the presence of a so-called weekend effect. Weekend admission has been associated with increased short-term mortality for intracerebral hemorrhage, ${ }^{12}$ but not subarachnoid hemorrhage. ${ }^{11}$ This may reflect the natural history of the disease process rather than differences in quality of care..$^{20}$

One large population of neurosurgical patients that our study did not include is trauma. At our institution, the majority of neurotrauma patients are managed by the trauma surgery service. The only trauma patients admitted to the neurosurgery service are those with isolated neurotrauma; those patients were included in this study. However, the polytrauma patients are all managed by the trauma surgery service. In that group of patients, ICU and hospital LOS is greatly impacted by other factors such as delivery of care by non-neurosurgery residents and other injuries or problems that may dictate hospital course. Another potential source of bias left unaddressed is the impact of multidisciplinary care on LOS.

The interpretation of our study's results is limited by our chosen study design. Our study was intended to demonstrate correlation, not specifically causation. However, our results are hypothesis generating and require validation in future prospective studies. Given these factors, it is unclear whether the findings from this single-institution study are generalizable to other residency training programs. Distribution of resident service coverage, protocols for regulating service handoffs, and degree of faculty oversight are all expected to differ, perhaps drastically, among institutions. Validation of these findings by multicenter studies is therefore recommended.

\section{Conclusions}

Transitions in care have the potential to negatively impact patient outcomes. We report that resident handoffs are associated with increased hospital and ICU LOS. The Patient assessment, Assertive communication, Continuum of care, Teamwork with trust (PACT) Project found that 
resident handoffs are largely unstructured and fail to adequately convey key patient information. ${ }^{32}$ Strategies for improving the quality of resident handoffs include minimizing distractions, using a standardized communication tool, and deliberate practice. ${ }^{3}$ Improved handoffs may mitigate some of the impacts on patient care observed in this study. A randomized controlled study on handoffs, specifically in surgical disciplines, is necessary to definitively establish the relationship between handoffs and outcomes such as mortality and hospital LOS. Future studies should also prospectively analyze the impact of handoffs on rates of postoperative complications, hospital-acquired conditions, and readmission rates.

\section{Acknowledgments}

This work was supported by a grant from the Kaul Foundation and the University of Alabama at Birmingham Center for Clinical and Translational Science (UL1 TR000165).

\section{References}

1. Accreditation Council for Graduate Medical Education: ACGME Common Program Requirements. (https://www. acgme.org/acgmeweb/Portals/0/PFAssets/Program Requirements/CPRs2013.pdf) [Accessed October 8, 2015]

2. Ahmed N, Devitt KS, Keshet I, Spicer J, Imrie K, Feldman L, et al: A systematic review of the effects of resident duty hour restrictions in surgery: impact on resident wellness, training, and patient outcomes. Ann Surg 259:1041-1053, 2014

3. Anderson CE, Nicksa GA, Stewart L: Distractions during resident handoffs: incidence, sources, and influence on handoff quality and effectiveness. JAMA Surg 150:396-401, 2015

4. Babu MA, Nahed BV, Heary RF: Investigating the scope of resident patient care handoffs within neurosurgery. PLoS One 7:e41810, 2012

5. Babu R, Thomas S, Hazzard MA, Friedman AH, Sampson JH, Adamson C, et al: Worse outcomes for patients undergoing brain tumor and cerebrovascular procedures following the ACGME resident duty-hour restrictions. J Neurosurg 121:262-276, 2014

6. Babu R, Thomas S, Hazzard MA, Lokhnygina YV, Friedman $\mathrm{AH}$, Gottfried ON, et al: Morbidity, mortality, and health care costs for patients undergoing spine surgery following the ACGME resident duty-hour reform: clinical article. J Neurosurg Spine 21:502-515, 2014

7. Billingsley P: Probability and Measure. Hoboken, NJ: Wiley, 2012

8. Blecker S, Shine D, Park N, Goldfeld K, Braithwaite RS, Radford MJ, et al: Association of weekend continuity of care with hospital length of stay. Int J Qual Health Care 26:530-537, 2014

9. Bollschweiler E, Krings A, Fuchs KH, Pistorius G, Bein T, Otto U, et al: Alternative shift models and the quality of patient care. An empirical study in surgical intensive care units. Langenbecks Arch Surg 386:104-109, 2001

10. Burton MC, Kashiwagi DT, Kirkland LL, Manning D, Varkey P: Gaining efficiency and satisfaction in the handoff process. J Hosp Med 5:547-552, 2010

11. Crowley RW, Yeoh HK, Stukenborg GJ, Ionescu AA, Kassell NF, Dumont AS: Influence of weekend versus weekday hospital admission on mortality following subarachnoid hemorrhage. Clinical article. J Neurosurg 111:60-66, 2009

12. Crowley RW, Yeoh HK, Stukenborg GJ, Medel R, Kassell NF, Dumont AS: Influence of weekend hospital admission on short-term mortality after intracerebral hemorrhage. Stroke 40:2387-2392, 2009
13. Dumont TM, Rughani AI, Penar PL, Horgan MA, Tranmer BI, Jewell RP: Increased rate of complications on a neurological surgery service after implementation of the Accreditation Council for Graduate Medical Education work-hour restriction. J Neurosurg 116:483-486, 2012

14. Gakhar B, Spencer AL: Using direct observation, formal evaluation, and an interactive curriculum to improve the sign-out practices of internal medicine interns. Acad Med 85:1182-1188, 2010

15. Ghaferi AA, Birkmeyer JD, Dimick JB: Complications, failure to rescue, and mortality with major inpatient surgery in Medicare patients. Ann Surg 250:1029-1034, 2009

16. Ghaferi AA, Birkmeyer JD, Dimick JB: Variation in hospital mortality associated with inpatient surgery. $\mathbf{N}$ Engl J Med 361:1368-1375, 2009

17. Gopaldas RR, Chu D, Dao TK, Huh J, LeMaire SA, Coselli JS, et al: Impact of ACGME work-hour restrictions on the outcomes of coronary artery bypass grafting in a cohort of 600,000 patients. J Surg Res 163:201-209, 2010

18. Grady MS, Batjer HH, Dacey RG: Resident duty hour regulation and patient safety: establishing a balance between concerns about resident fatigue and adequate training in neurosurgery. J Neurosurg 110:828-836, 2009

19. Grinstead CM, Snell JL: Introduction to Probability, ed 2. Providence, RI: American Mathematical Society, 1997

20. Heros RC: Weekend versus weekday admission mortality. J Neurosurg 111:57-59, 2009

21. Hoh BL, Neal DW, Kleinhenz DT, Hoh DJ, Mocco J, Barker FG II: Higher complications and no improvement in mortality in the ACGME resident duty-hour restriction era: an analysis of more than 107,000 neurosurgical trauma patients in the Nationwide Inpatient Sample database. Neurosurgery 70:1369-1382, 2012

22. Hsiao AL, Shiffman RN: Dropping the baton during the handoff from emergency department to primary care: pediatric asthma continuity errors. Jt Comm J Qual Patient Saf 35:467-474, 2009

23. Jagannathan J, Vates GE, Pouratian N, Sheehan JP, Patrie J, Grady MS, et al: Impact of the Accreditation Council for Graduate Medical Education work-hour regulations on neurosurgical resident education and productivity. J Neurosurg 110:820-827, 2009

24. Jagsi R, Kitch BT, Weinstein DF, Campbell EG, Hutter M, Weissman JS: Residents report on adverse events and their causes. Arch Intern Med 165:2607-2613, 2005

25. Jamal MH, Doi SA, Rousseau M, Edwards M, Rao C, Barendregt JJ, et al: Systematic review and meta-analysis of the effect of North American working hours restrictions on mortality and morbidity in surgical patients. Br J Surg 99:336-344, 2012

26. Kaderli R, Businger A, Oesch A, Stefenelli U, Laffer U: Morbidity in surgery: impact of the 50-hour work-week limitation in Switzerland. Swiss Med Wkly 142:w13506, 2012

27. Lofgren RP, Gottlieb D, Williams RA, Rich EC: Post-call transfer of resident responsibility: its effect on patient care. $\mathbf{J}$ Gen Intern Med 5:501-505, 1990

28. Norby K, Siddiq F, Adil MM, Haines SJ: The effect of duty hour regulations on outcomes of neurological surgery in training hospitals in the United States: duty hour regulations and patient outcomes. J Neurosurg 121:247-261, 2014

29. Nouraei SA, Hudovsky A, Frampton AE, Mufti U, White NB, Wathen CG, et al: A study of clinical coding accuracy in surgery: implications for the use of administrative big data for outcomes management. Ann Surg 261:1096-1107, 2015

30. Riesenberg LA, Leitzsch J, Massucci JL, Jaeger J, Rosenfeld JC, Patow C, et al: Residents' and attending physicians' handoffs: a systematic review of the literature. Acad Med 84:1775-1787, 2009

31. Schuberth JL, Elasy TA, Butler J, Greevy R, Speroff T, Dit- 
tus RS, et al: Effect of short call admission on length of stay and quality of care for acute decompensated heart failure.

Circulation 117:2637-2644, 2008

32. Tapia NM, Fallon SC, Brandt ML, Scott BG, Suliburk JW: Assessment and standardization of resident handoff practices: PACT project. J Surg Res 184:71-77, 2013

33. Thaller M, Hughes T: Inter-rater agreement of observable and elicitable neurological signs. Clin Med 14:264-267, 2014

34. Van Eaton EG, Horvath KD, Lober WB, Rossini AJ, Pellegrini CA: A randomized, controlled trial evaluating the impact of a computerized rounding and sign-out system on continuity of care and resident work hours. J Am Coll Surg 200:538-545, 2005

35. Yeung L, Miraflor E, Garcia A, Victorino GP: Effect of surgery resident change of shift on trauma resuscitations and outcomes. J Surg Educ 70:87-94, 2013

\section{Disclosures}

The authors report no conflict of interest concerning the materials or methods used in this study or the findings specified in this paper.

\section{Author Contributions}

Conception and design: Kuhn, Davis, Markert. Acquisition of data: Kuhn, Davis. Analysis and interpretation of data: Kuhn, Davis. Drafting the article: Kuhn, Davis. Critically revising the article: all authors. Reviewed submitted version of manuscript: all authors. Approved the final version of the manuscript on behalf of all authors: Kuhn. Statistical analysis: Kuhn, Agee, Oster. Study supervision: Markert.

\section{Supplemental Information}

Previous Presentations

Portions of this work were presented as an oral abstract presentation at the 66th Annual Meeting of the Southern Neurosurgical Society, Naples, Florida, March 26, 2015.

\section{Correspondence}

Elizabeth N. Kuhn, Department of Neurosurgery, University of Alabama at Birmingham, 1720 Second Ave. S, FOT 1062, Birmingham, AL 35294-3410. email: ekuhn@uabmc.edu. 